\title{
Uma breve revisão bibliográfica sobre a exergia em refrigeradores
}

\author{
A short literature review of exergy in refrigerators \\ Una breve revisión bibliográfica sobre la exergía en los refrigeradores
}

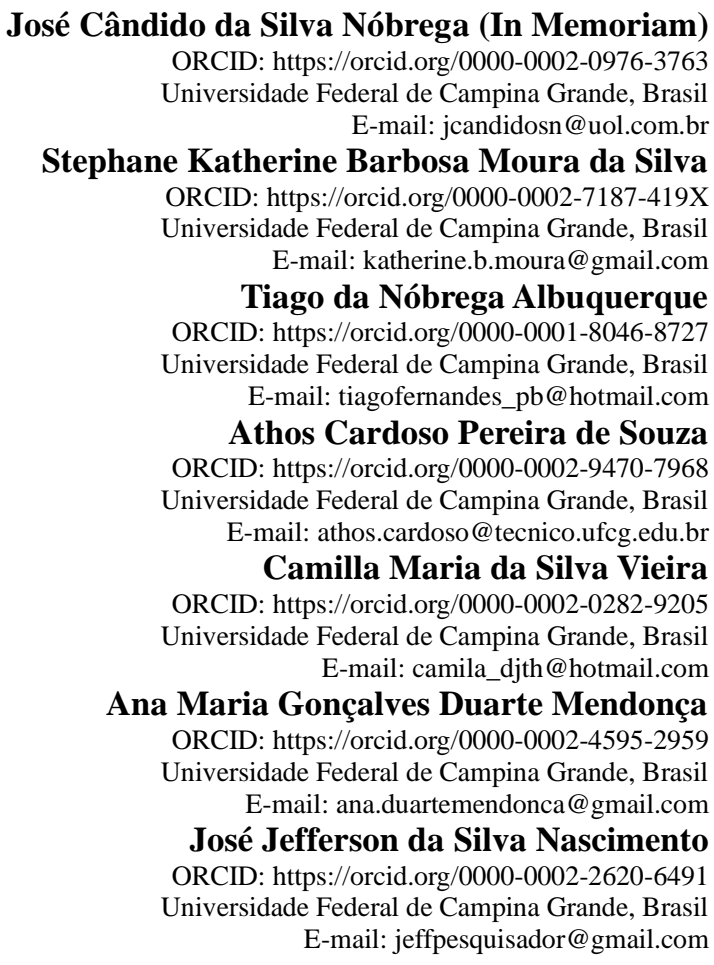

\section{Resumo}

A energia, apresentada em diversas formas, é de fundamental importância para o desenvolvimento da humanidade e, sua correta compreensão para o potencial com posterior conversão para auxiliar nas atividades laborais sempre foi desafio e motivação para as mentes mais brilhantes. Logo, é fundamental investigar maneiras de melhorar a eficiência no uso de recursos energéticos destaca-se análise exergética, no qual em sido aplicada desde os anos 1970, com finalidade de encontrar mais utilização racional da energia, e, ao mesmo tempo, reduzir o consumo de combustíveis fósseis e transformando a aplicação de eficiência energética. Neste contexto, o presente artigo tem como objetivo realizar uma revisão bibliográfica sobre exergia em refrigeradores. Verificou-se que o objetivo do trabalho foi cumprido, tendo em vista que foi apresentado 9 estudos que verificaram a eficiência energética por meio a exergia em refrigeradores. Constatou-se poucos estudos da literatura brasileira, apresentando-se como uma carência.

Palavras-chave: Eficiência; Energia; Exergia; Refrigeradores; Termodinâmica.

\begin{abstract}
Energy, presented in various forms, is of fundamental importance to the development of mankind, and its correct understanding for the potential with subsequent conversion to assist in work activities has always been a challenge and motivation for the brightest minds. Therefore, it is essential to investigate ways to improve the efficiency in the use of energy resources, especially exergetic analysis, which has been applied since the 1970s, in order to find more rational use of energy, and, at the same time, reduce the consumption of fossil fuels and transforming the application of energy efficiency. In this context, the present paper aims to perform a literature review on exergy in refrigerators. It was verified that the objective of the work was met, since it was presented 9 studies that verified the energy efficiency through exergy in refrigerators. It was found that there are few studies in the Brazilian literature, presenting itself as a lack.
\end{abstract}

Keywords: Efficiency; Energy; Exergy; Refrigerators; Thermodynamics. 


\begin{abstract}
Resumen
La energía, que se presenta en diversas formas, es de fundamental importancia para el desarrollo de la humanidad y, su correcta comprensión para el potencial con posterior conversión para ayudar en las actividades laborales, siempre ha sido un reto y motivación para las mentes más brillantes. Por lo tanto, es fundamental investigar formas de mejorar la eficiencia en el uso de los recursos energéticos, destacando el análisis exergético, que se viene aplicando desde la década de 1970, con el fin de encontrar un uso más racional de la energía, y al mismo tiempo, reducir el consumo de combustibles fósiles y transformar la aplicación de la eficiencia energética. En este contexto, el presente artículo pretende realizar una revisión bibliográfica sobre la exergía en los frigoríficos. Se verificó que el objetivo del trabajo se cumplió, considerando que se presentaron 9 estudios que verificaron la eficiencia energética a través de la exergía en refrigeradores. Se encontró que hay pocos estudios en la literatura brasileña, presentándose como una carencia.

Palabras clave: Eficiencia; Energía; Exergía; Refrigeradores; Termodinámica.
\end{abstract}

\title{
1. Introdução
}

Desde os primórdios da revolução industrial, a humanidade tem contado cada vez mais com máquinas para a realização de trabalho e de uma parte cada vez maior da produção. As máquinas foram responsáveis por permitir ganhos significativos de produtividade às atividades humanas. Entre as principais máquinas utilizadas, encontram-se os refrigeradores, no qual os mais antigos utilizam um gás que é um dos responsáveis por deixar as temperaturas do nosso planeta cada vez mais altas, conhecido como HFC, responsável pelo resfriamento de sistemas (Lugo-Leyte et al., 2013).

A energia, apresentada em diversas formas, é de fundamental importância para o desenvolvimento da humanidade e, sua correta compreensão para o potencial com posterior conversão para auxiliar nas atividades laborais sempre foi desafio e motivação para as mentes mais brilhantes. Em um contexto mundial de escassez de recursos energéticos, especialmente os de origem fóssil, e em um cenário de cada vez maior conscientização do problema das mudanças climáticas causado pela emissão de gases de efeito estufa (como o dióxido de carbono), é fundamental investigar maneiras de melhorar a eficiência no uso de recursos energéticos (Heidinger, 2018).

Destaca-se análise exergética, no qual em sido aplicada desde os anos 1970, com finalidade de encontrar mais utilização racional da energia, e, ao mesmo tempo, reduzir o consumo de combustíveis fósseis e transformando a aplicação de eficiência energética (Silva; Junior; Costa, 2012). Faria (2014) afirma que o conceito de exergia foi introduzido há dois séculos quando Carnot (1824), desenvolveu o primeiro trabalho que estabeleceu as bases da Segunda Lei da Termodinâmica, através da ideia de quantificar o trabalho máximo que podia ser obtido nas máquinas de vapor. Os primeiros enunciados da Segunda Lei da Termodinâmica foram apoiados por Clausius (1850) e Kelvin e Planck (1897), e mais posteriormente por Carathéodory (1909).

Os processos energéticos são apresentados por duas leis fundamentais: a primeira e segunda lei da termodinâmica. A primeira lei, fundamentado no princípio da conservação da energia, afirma que a quantidade de energia transita no sistema através de fluxo de massa, transferência de calor e trabalho. A segunda lei, por sua vez, leva em consideração a parcela de ordem de desordem, como exemplo a irreversibilidades. Sendo assim, a energia também apresenta qualidade, e os processos reais ocorrem na direção do aumento da entropia e da diminuição da qualidade da energia. A junção dessas duas leis resulta no conceito de "exergia" (Silva et al., 2018).

O esforço de um sistema é definido como o máximo trabalho de eixo que pode ser feito pelo composto do sistema e um ambiente de referência especificado. O ambiente de referência é infinito, em equilíbrio, e para abranger todos os outros sistemas. Caracteristicamente, o ambiente é especificado pela indicação de sua temperatura, pressão e composição química. A exergia não é apenas uma propriedade termodinâmica; é uma propriedade tanto de um sistema quanto do ambiente de referência (Jantar \& Rosen, 2012). O conceito de exaustão apresenta o potencial máximo de um sistema para realizar trabalhos é uma função de sua energia interna e das condições ambientais (pressão, temperatura e composição).

O termo exergia vem das palavras gregas ex e ergonômico, significando de e trabalho. A exergia de um sistema pode 
ser aumentada se a exergia for entrada (por exemplo, o trabalho é feito sobre ela) (Jantar \& Rosen, 2012). Silva, Junior e Costa (2012) definem exergia como o maior trabalho teórico possível de ser obtido segundo o ambiente de referência interaja até o equilíbrio com o sistema de interesse. Ou seja, é uma medida do potencial de um fluxo de energia para ser transformada em energia de alta qualidade. $\mathrm{O}$ seu estudo tem como objetivo descobrir mais utilização racional da energia, reduzindo o consumo de combustíveis fósseis e transformando a aplicação de eficiência energética.

Neste sentido, o presente artigo tem como objetivo realizar uma revisão bibliográfica sobre exergia em refrigeradores, buscando apresentar os principais estudos relacionados a eficiência energética a partir do máximo trabalho de eixo que pode ser feito pelo composto do sistema e um ambiente de referência especificado. A presente pesquisa justifica-se pela necessidade de fomentar esse assunto na literatura, visto que é um tema com poucos trabalhos publicados na literatura nacional.

\section{Referencial Teórico}

\subsection{Exergia}

A busca de padrões de qualidade termodinâmica é um dos desafios mais interessantes da Termodinâmica de Engenharia. Nas últimas décadas, esta preocupação não se restringe mais à quantificação do desempenho de um determinado processo ou equipamento, mas se estende a todos os limites de qualquer processo de conversão de energia, incluindo suas entradas e desperdícios energéticos (Oliveira Junior, 2012).

Logo, a exergia é uma medida do potencial de um fluxo para causar mudanças, como consequência de não ser completamente estável em relação ao ambiente de referência. Além disso, não está sujeita a uma lei de conservação; em vez disso, é consumida ou destruída, devido a irreversibilidades em qualquer processo, bem como não pode ser perdida, como afirmado na primeira lei da termodinâmica (Araújo et al., 2019). "A exergia está sempre envolvida nos processos onde se operam conversões de energia, como: a irreversibilidade dos processos reais; a transformação de energia térmica em trabalho (energia organizada); a indicação do limite até onde a energia pode ser útil” (Gonçalves \& Gaspar, 2011, p. 2).

Segundo Albuquerque (2017), para utilizar de forma adequada os conceitos da exergia, usam-se os parâmetros ambientais como o estado de referência (estado morto), em que significa que está em equilíbrio termodinâmico com o ambiente em que se encontra. No estado morto, um sistema está na temperatura e pressão de seu meio ambiente; ele não tem energia cinética ou potencial relativo ao ambiente; e ele não reage com o ambiente. Além disso, não há desbalanceamento magnético, efeitos elétricos e de tensão superficial entre o sistema e sua vizinhança se estes são relevantes para situação em mãos. Logo, as propriedades do sistema no estado morto são denotadas com o subscrito zero, por exemplo, $\mathrm{P}=0, \mathrm{~T}=0, \mathrm{~h}=0$, $\mathrm{u}=0$, e $\mathrm{s}=0$.

Neste sentido, quando um sistema sofre um processo inteiramente reversível até atingir o estado em que esteja em equilíbrio com o meio, o sistema terá realizado o máximo de trabalho possível. Logo, é possível definir a disponibilidade de um estado em função da capacidade (potencial) para realizar o máximo de trabalho possível. Admitindo um processo em regime permanente para um volume de controle que apresenta uma única seção de entrada e uma única seção de saída (Almeida; Barbosa; Fontes, 2010). Neste sentido, os autores afirmam que o trabalho reversível é fornecido pela Equação 1.

$$
w_{v c}^{r e v}=\left(h_{t o t, e}-t 0_{s e}\right)-\left(h_{t o t, s}-t 0_{s s}\right)+\sum j \neq 0\left(1-\frac{T_{0}}{T_{j}}\right) q_{v c . j}
$$


Almeida, Barbosa e Fontes (2010) afirmam que o termo q_(vc.j) se refere a contribuição das transferências de calor dos reservatórios ao trabalho líquido reversível. Esta transferência de disponibilidade proporciona o potencial para a realização de trabalho às máquinas térmicas e que esta contribuição está separada das disponibilidades associadas ao escoamento do fluido de trabalho. O trabalho reversível será máximo quando a massa, que deixa o volume de controle, estiver em equilíbrio com o meio. Logo, se considerar esse estado pelo índice 0 , o trabalho reversível será máximo quando hs $=\mathrm{h} 0$; $\mathrm{ss}=\mathrm{s} 0$; Vs $=0$ $\mathrm{zs}=\mathrm{z}$. Afirma-se que o trabalho reversível máximo é definido pela Equação 2.

$\psi=\left(h+\frac{v^{2}}{2}+g z-t 0_{s}\right)-\left(h_{0}+g z_{0}-t o_{s 0}\right)$

No entanto, a definição matemática de exergia é apresentada na Equação 3, no qual é obtida a partir da aplicação de balanços de energia e de entropia a um sistema interagindo com o meio ambiente, em que os símbolos E (U+EC+EP), $\square$ e S denotam respectivamente, a energia, o volume e a entropia do sistema num estado específico e U0, $\square 0$ e S0 são os valores das mesmas propriedades se o sistema estivesse no estado morto (Albuquerque, 2017).

$$
X=\left(E-U_{0}\right)+\left(\forall-\forall_{0}\right)-T_{0}\left(S-S_{0}\right)
$$

\subsection{Refrigeradores}

Os dispositivos que promovem refrigeração são conhecidos como refrigeradores e eles funcionam em ciclos de refrigeração. O ciclo de refrigeração mais habitualmente usado na atualidade é o ciclo de refrigeração por compressão a vapor, em que o fluido refrigerante é condensado e vaporizado alternadamente e é comprimido na fase de vapor (Miranda, 2017). O ciclo resultante é chamado de ciclo ideal de refrigeração por compressão de vapor (Figura 1).

Figura 1: Diagramas de refrigeração e T-s do ciclo ideal.
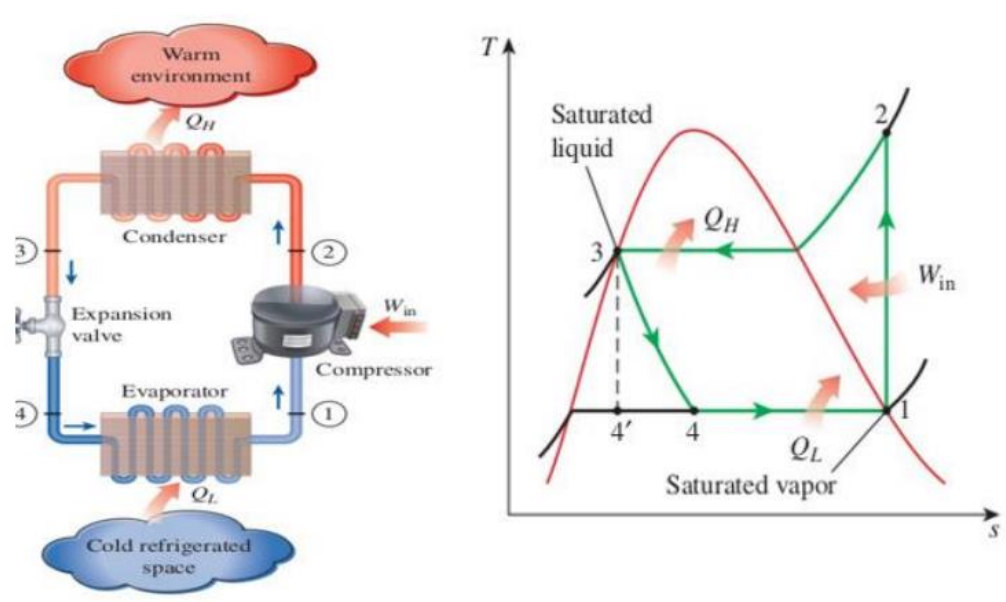

Fonte: Çengel e Boles (2015).

O ciclo padrão de refrigeração por compressão de vapor passa por quatro processos básicos (Figura 2). No entanto, pode haver sistemas mais complexos, como os ciclos de múltiplos estágios de compressão, no qual envolvem um número maior de componentes, mas o princípio básico de funcionamento é o mesmo (Toigo, 2013). 
Figura 2: Ciclo padrão por compressão de vapor.

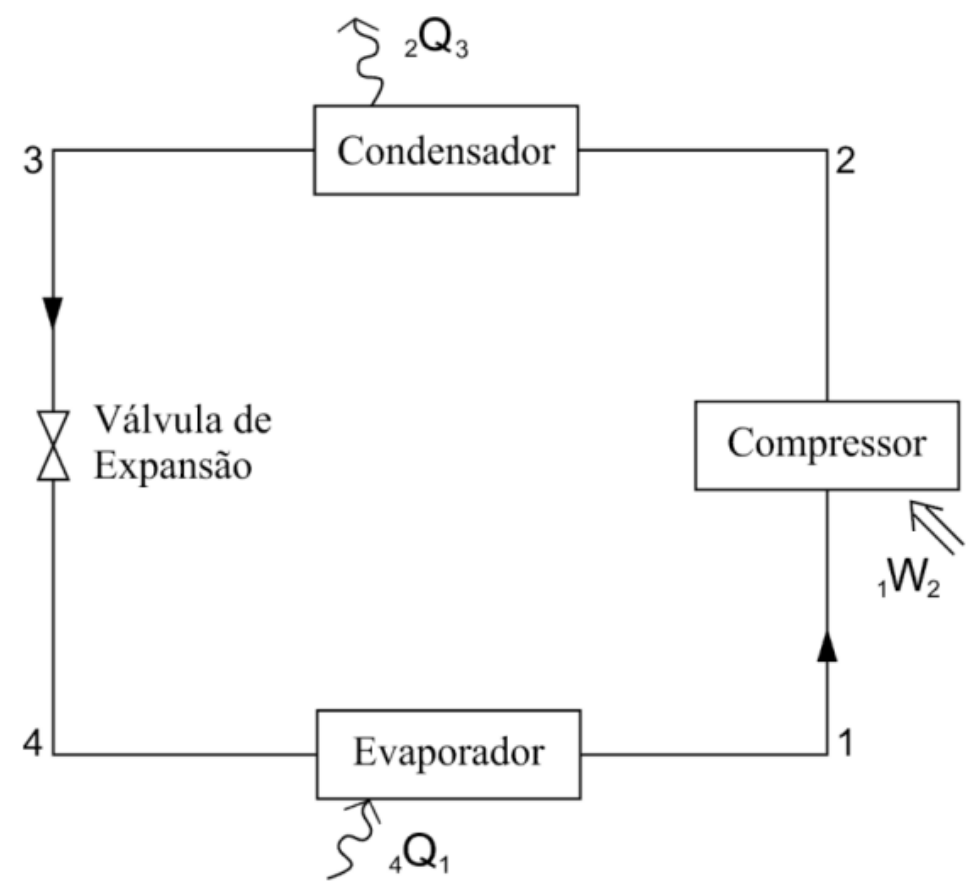

Fonte: Jones (2007).

Toigo (2013) afirma que o fluido refrigerante circunda entre os elementos e se responsabiliza por deslocar o calor captado no evaporador, até o meio externo por meio do condensador. No evaporador, o fluido está à baixa pressão e temperatura, a fim de que receba calor do meio. Posteriormente, o fluido tem sua pressão e temperatura aumentada, pelo trabalho do compressor. O calor recebido até então é rejeitado no condensador e, depois, o fluido refrigerante volta a ter sua temperatura e pressão reduzida através da válvula de expansão

\section{Metodologia}

\subsection{Tipo da Pesquisa}

Conforme caracteriza Köche (2016), do ponto de vista da natureza, esse trabalho trata de uma pesquisa básica, também conhecida como pesquisa fundamental, focada em ampliar o conhecimento que temos do mundo e tudo o que o forma. Esse tipo de pesquisa também é conhecido como fundamental, visto que objetiva acender conhecimentos para a ciência sem que estes tenham uma aplicação prática.

Pela perspectiva de abordagem, é uma pesquisa qualitativa, ou seja, é desenvolvido conceitos, ideias e entendimentos através de padrões encontrados nos dados, ao invés de coletar dados para comprovar teorias, hipóteses e modelos preconcebidos (Cardano, 2017). Analisando os objetivos essa pesquisa é exploratória, ou seja, a finalidade é proporcionar maior familiaridade com o problema, tornar-se explicito ou construir hipóteses com seu respeito ou causar aprimoramento do tema (Neto, 2017). Em relação aos procedimentos técnicos, é do tipo Revisão Bibliográfica, no qual proporciona uma síntese de conhecimento e a incorporação dos resultados de estudos significativos, fundamentado em diversas pesquisas já publicadas.

\subsection{Realização do estudo}

Para a realização desse estudo, a primeira etapa foi a organização do problema a ser pesquisado, para posteriormente avaliar e aplicar todo o máximo do material bibliográfico disponível, uma vez que o tema deve conter relevância tanto teórica como prática e proporcionar interesse de ser estudado. 
O presente estudo foi realizado com base em uma revisão bibliográfica, utilizando trabalhos científicos, acerca do Jornalismo negro no Brasil, através de dados da literatura científica Scielo e a Plataforma de Pesquisa Google Acadêmico e sites especializados no assunto, tendo as seguintes palavras-chave: "Energia", "Exergia", "Termodinâmica" e "Refrigeradores".

Os dados foram obtidos através de publicações em Revistas Científicas, Trabalhos de Conclusão de Curso, Dissertações de Mestrado e Tese de Doutorado. Para organizar as informações dos trabalhos selecionados da base de dados, foi utilizada a leitura flutuante dos títulos e resumos dos trabalhos bem como os resultados apresentados.

Para organizar as informações dos trabalhos selecionados da base dados, foi utilizada a leitura flutuante dos títulos e resumos dos trabalhos bem como os resultados apresentados. O principal critério de exclusão de artigos está relacionado ao tempo da publicação, visto que apenas obras mais recentes são bem vistas no meio cientifico. A ordem de prioridade para a escolha de trabalho foi: (i) artigos publicados em periódicos internacionais; (ii) artigos publicados em periódicos nacionais reconhecidos; (iii) livros publicados por bons editores; (iv) teses e dissertações; (v) anais de conferências internacionais; (vi) anais de conferências nacionais.

Critérios de inclusão: estudos encontrados na base de dado escolhida, publicados em período indeterminado, utilizando os descritores já citados. Critérios de exclusão: artigo noticiosos, textos em resenhas, artigos não indexados, opiniões, editoriais ou manuais. Para organizar as informações dos trabalhos selecionados da base dados, foi utilizada a leitura flutuante dos títulos e resumos dos trabalhos, bem como os resultados apresentados

\section{Resultados e Discussão}

Tirandazi et al (2011), em um artigo com finalidade de realizar uma análise exergética do ciclo de refrigeração de vários estágios usado para Etano e hidrocarbonetos mais pesados $(C 2+)$, os autores identificaram que usando um valor de entrada de trabalho real típico; a eficiência exergética do ciclo de refrigeração é determinada em 43,45\%, indicando um grande potencial de melhorias. Os resultados da simulação revelaram que as eficiências exergéticas do trocador de calor e das seções de expansão obtêm a classificação mais baixa entre os outros compartimentos do ciclo de refrigeração. Cálculos de refrigeração foram realizados através da análise de temperatura-entropia (Diagramas T - S ) e pressão-entalpia ( P - H ) onde o coeficiente de desempenho (COP) foi obtido como 1,87. A novidade deste artigo inclui o efeito e a análise de sensibilidade da queda de pressão e temperatura sobre a eficiência exergética e coeficiente de desempenho do ciclo.

$\mathrm{Xu}$ (1992) determinou experimentalmente as perdas exergéticas nos componentes de refrigeradores e freezers operando com CFC 12, HFC 134a e HC 290 e encontrou em todos os casos a maior taxa de destruição de exergia no compressor. Com finalidade de descreve uma análise de esforço em um sistema de refrigeração por compressão de vapor, Xu e Clodic (1992) desenvolveram um modelo matemático para a realização de análise de esforço por computador de uma experiência e uma análise de esforço em 3 geladeiras/congeladores usando R12, R134a e R290. O autor determinou experimentalmente as perdas exergéticas nos componentes de refrigeradores e freezersoperando com CFC 12, HFC 134a e HC 290 e encontrou em todos os casos a maior taxa de destruição de exergia no compressor. Xu e Clodic (1992) verificaram que a maior parte das perdas por esforço é em compressor e em evaporador para este sistema de compressão de vapor. O refrigerador do R134a é quase eficiente do que o do R12. Mas os refrigeradores RI34a e R290 são menos eficientes que o refrigerador R12. Cada um tem algumas dificuldades para conseguir o mesmo desempenho do R12.

Nikolaidis e Probert (1998) com objetivo de realizar uma análise pelo método de exergia do desempenho de instalações de refrigeração por compressão de vapor em dois estágios, verificaram que, quanto maiores são as diferenças entre as temperaturas do condensador e do ambiente, e do evaporador e da câmara fria, as taxas de irreversibilidades são maiores. Os pesquisadores chegaram à conclusão que as reduções destas diferenças de temperatura contribuem para a redução das 
irreversibilidades totais da planta. Ou seja, as irreversibilidades das compressões são as predominantes.

Santos e Costa Filho (2018), com finalidade de analisar e comparar o desempenho de refrigeradores, funcionando resfriando ou aquecendo o ar de um ambiente fechado na cidade do Rio de Janeiro, verificaram que o CFC 12 apresenta melhor desempenho do que o HFC 134a pelas $1^{\mathrm{a}}$. e $2^{\mathrm{a}}$. Leis, sendo os desempenhos de ambos significativamente superior ao do HCFC 22. Deste modo, o agente refrigerante melhor do ponto de vista ambiental possui desvantagens de desempenho em relação àquele com uso proibido.

Em uma pesquisa com intuito de realizar uma otimização exergética de sistema de refrigeração por absorção, Souza (2020) realizou uma análise paramétrica com base na variação dos parâmetros operacionais do sistema como temperatura, pressão e fração de massa de amônia, em que foi possível entender o impacto de tais parâmetros na eficiência exergética. Foi realizada uma análise exergética do sistema com o intuito de obter quais componentes são responsáveis pela maior parcela da destruição de exergia total. Para o refrigerador modelado, o autor verificou que o gerador e o absorvedor são as maiores fontes de irreversibilidades do sistema. Além do mais, foram obtidos pontos ótimos de eficiência exergética e redução da destruição de exergia no gerador/retificador em função de valores de temperatura e pressão em determinadas condições operacionais.

Albuquerque (2017), em uma análise energética e exergética de uma unidade de refrigeração, o pesquisador buscou avaliar os comportamentos energético e exergético, fundamentados na primeira lei e na segunda lei da termodinâmica, no qual foi possível avaliar o comportamento do equipamento com a mudança de alguns dos parâmetros normais de trabalho, como pressões de condensação e evaporação, temperaturas de condensação e evaporação e fluido refrigerante. Os resultados mostraram que o desempenho da unidade melhora ao diminuir a variação de pressão entre o condensador e o evaporador e que as eficiências energéticas e exegética sofrem um acréscimo com o aumento da temperatura de evaporação e uma redução com a elevação da temperatura de condensação.

Yumrutas et al. (2002) realizaram uma análise exergética de um ciclo de refrigeração operando com amônia. Os pesquisadores verificaram que as temperaturas de evaporação e condensação têm fortes efeitos sobre as perdas de esforço no evaporador e condensador, e sobre a segunda lei de eficiência e COP do ciclo, mas poucos efeitos sobre as perdas de esforço no compressor e na válvula de expansão. A segunda lei de eficiência e o COP aumenta, e a perda total de esforço diminui com a diminuição da diferença de temperatura entre o evaporador e o espaço refrigerado e entre o condensador e o ar externo.

Em uma pesquisa com finalidade de realizar uma comparação do desempenho energético e exergético de um sistema de refrigeração por compressão de vapor usando refrigerantes hidrocarboneto puro (HC), Bayrakçi e Özgür (2009) usaram quatro diferentes HCs propano puro (R290), butano (R600), isobutano (R600a) e isopentano (R1270). Os resultados apresentaram as diferenças dos valores de coeficiente de desempenho de resfriamento desses refrigerantes, no qual foram bastante pequenas. Os valores de eficiência energética e exergética obtidos com R1270 e R600 foram superiores a R600a e R290.

Chandrasekharan (2014), realizou uma análise comparativa da influência do refrigerante sobre o desempenho de um simples' vapor sistema de refrigeração por compressão. O estudo é baseado nos refrigerantes R12 e R134a. O autor verificou que a eficiência exergética aumenta com o grau de sub-resfriamento para ambos os refrigerantes, no entanto, a variação foi mais acentuada para o R134a que para o R12. As Eficiências exergeticas de ambos os refrigerantes diminuem com o aumento do evaporador temperatura. Em temperaturas de evaporador mais baixas, o A eficiência da VCRS operando no R134a é maior do que aquelas operando no R12. Mas em temperaturas de evaporador mais altas, R12 tem maior eficiência de esforço do que o sistema R134a. 


\section{Considerações Finais}

O presente artigo buscou realizar uma revisão bibliográfica sobre exergia em refrigeradores, buscando apresentar os principais estudos relacionados a eficiência energética a partir do máximo trabalho de eixo que pode ser feito pelo composto do sistema e um ambiente de referência especificado.

Logo, verifica-se que o objetivo do trabalho foi cumprido, tendo em vista que foi apresentado 9 estudos que verificaram a eficiência energética por meio a exergia em refrigeradores. Verificou-se poucos estudos da literatura brasileira, apresentando-se como uma carência. Neste sentido, recomenda-se para futuras pesquisas estudos semelhantes a presente pesquisa para diferentes fluidos refrigerantes. Sendo assim, espera-se que a presente pesquisa contribua com a literatura cientifica e sirva de base para futuras pesquisas sobre o tema.

\section{Agradecimentos}

Os autores agradecem às Agências Financiadoras, como CAPES, CNPq, FAPESQ, pelo apoio financeiro e, dedicam in memoriam, ao colega José Cândido da Nóbrega.

\section{Referências}

Albuquerque, C. E. S. (2017). Avaliação do comportamento energético e exergético de um sistema de refrigeração por compressão de vapor. estudo de caso: resfriamento e congelamento de banana. 2017. 150 f. Dissertação (Mestrado em Engenharia Mecânica) -Universidade Federal Campina Grande.

Araujo, A. A. Q., Nascimento, R. S., \& Oliveira, R. (2019). Exergia e meio ambiente. IN: Anais do cCngresso Brasileiro de Gestão Ambiental e Sustentabilidade, 7 (1), 401-406.

Assunção, G. H., Reis, M. A. A., \& Abreu, M. C. S. (2016). Disgrafia, discalculia e dislexia: suas implicações na educação infantil. Linha de Pesquisa: 5a Estudos Culturais e Linguagens na Educação, 748.

Bayrakçi, H. C., \& Özgür, A. E. (2009). Análise de energia e exergia de sistema de refrigeração por compressão de vapor usando refrigerantes de hidrocarboneto puro. International Journal of Energy Research, 33 (12), 1070-1075.

Cardano, M. (2017). Manual de pesquisa qualitativa. a contribuição da teoria da argumentação. Tradução: Elisabeth da Rosa Conill. Petrópolis, Vozes.

Çengel, A. Y., Boles, M. A. (2015). Properties of pure substances. Thermodynamics: an engineering approach, 111-162.

Fria, P. R. (2014). Uma avaliação das metodologias de desagregação da exergia física para a modelagem termoeconômica de sistemas. Dissertação de Mestrado - Universidade Federal do Espírito Santo.

Gil, A. C. et al. (2002). Como elaborar projetos de pesquisa. Atlas.

Gonçalves, L. C. C., \& Gaspar, P. D. (2011). Energia, entropia, exergia. IN: Congresso Internacional de Engenharia, 1 (1)1-12.

Heidinger, G. G. (2018). Estudos experimentais de análise do desempenho termodinâmico e eficiência energética de equipamentos de refrigeração em climas tropicais. 2018. 221f. Tese de Doutorado (Engenharia Mecânica) - Universidade da Beira Interior, Covilhã

Jantar, I, \& Rosen, M. (2012). Exergia: energia, meio ambiente e desenvolvimento sustentável. Newnes.

Jones, W. P. (2007). Engenharia de ar condicionado. Routledge.

Köche, J. C. (2016). Fundamentos de metodologia científica. Editora Vozes.

Lakatos, E. M., \& Marconi, M. A. (2017). Metodologia do trabalho científico. (8a ed.), Atlas.

Lugo-Leyte, R. et al. (2013). Análisis de costos de operación exergoeconómicos a un ciclo teórico de refrigeración por compresión de vapor usando hfc-134a. Revista Mexicana de Ingeniería Química, 12 (2), 361-370.

Miranda, S. F. T. M. (2017). Análise termodinâmica de um refrigerador funcionando com diferentes fluidos refrigerantes sintéticos. 2017. 52f. Dissertação (Mestrado em Engenharia Mecânica) - Universidade Federal do Rio Grande do Norte.

Nikolaidis, C., \& Probert, D. (1998). Análise pelo método de exergia do desempenho de instalações de refrigeração por compressão de vapor em dois estágios. Energia Aplicada, 60 (4), 241-256.

Oliveira Junior, S. (2012). Exergia: produção, custo e renovabilidade. Springer Science \& Business Media.

Praça, F. S. G. (2015). Metodologia da pesquisa científica: organização estrutural e os desafios para redigir o trabalho de conclusão. Revista Eletrônica Diálogos Acadêmicos, 8(1), 72-87. 
Research, Society and Development, v. 11, n. 2, e5111224026, 2022

(CC BY 4.0) | ISSN 2525-3409 | DOI: http://dx.doi.org/10.33448/rsd-v11i2.24026

Santos, H. C., \& Costa Filho, M. A. F. (2008). Análise termodinâmica de um equipamento de dupla função: refrigerador e bomba de calor. IN: Congresso Nacional de Engenharia Mecânica, 5(1), 1-10.

Santos, J. A., \& Parra Filho, D. (2012). Metodologia Científica. IN: Faveni.

Silva, S. C., Junior, E. C., \& Costa, A. (2012). Conceitos fundamentais da propriedade termodinâmica exergia e exemplos de aplicação para análise de processos reais. Enciclopédia Biosfera, 8(15).

Silva, S. R. et al. (2018). Aplicação da propriedade termodinâmica exergia na avaliação de processos de produção de etanol lignocelulósico: uma revisão. Revista Virtual de Química, (5).

Souza, G. D. G. (2020). Otimização exergética de sistema de refrigeração por absorção. 87 f. Dissertação de Mestrado (Fenômenos de Transporte e Mecânica dos Sólidos) - Universidade Federal do Paraná, 2020.

Tirandazi, B. et al. (2011). Análise exergética de ciclos de refrigeração de centrais de recuperação c2 +. Pesquisa e Projeto de Engenharia Química, 89 (6), 676-689.

Toigo, E. (2017). Desenvolvimento do projeto de uma bancada didática de refrigeração por compressão de vapor. 79 f. Trabalho de Conclusão de Curso Universidade de Caxias do Sul.

Xu, X., \& Clodic, D. (1992). Análise de exergia em um sistema de refrigeração por compressão de vapor usando r12, r134a e r290 como refrigerantes. IN: International Refrigeration and Air Conditioning Conferenc, 1-11.

Yumrutaş, R., Kunduz, M., \& Kanoğlu, M. (2002). Análise exergética de sistemas de refrigeração por compressão de vapor. Exergy, Um Jornal Internacional, 2(4)266-272. 